\title{
B-SSCT: A Block Sequential Spectrum Sensing Scheme for Cognitive Radio
}

\author{
Yan Xin, Honghai Zhang, Sampath Rangarajan, and Kyungtae Kim \\ Mobile Communications and Networking Research \\ NEC Laboratories America, Inc., Princeton, NJ 08540, USA \\ E-mail: \{yanxin, honghai, sampath, kyungtae\}@nec-labs.com
}

\begin{abstract}
Recently, it has been shown that in comparison to the well-known energy detection scheme, the sequential shifted chi-square test (SSCT) is capable of delivering considerable reduction on the average sample number (ASN) while maintaining a comparable detection error performance for spectrum sensing. Nonetheless, SSCT needs to perform threshold comparisons on every received sample, which may be difficult or even infeasible in practice particularly when the sampling rate is high and/or the signal-to-noise ratio is low. This paper proposes an extension of SSCT, called block-wise SSCT (B-SSCT), to overcome this shortcoming. Numerical algorithms are applied to evaluate the false-alarm and miss-detection probabilities and the ASN of B-SSCT, in a recursive fashion. Simulation and numerical results show that compared to the original SSCT, BSSCT is capable of achieving almost the same detection error performance with a significantly reduced number of threshold comparisons and a slightly increased ASN. An implementation example demonstrates potential practical feasibility of B-SSCT in a real environment.
\end{abstract}

\section{INTRODUCTION}

In a cognitive radio (CR) network, spectrum sensing plays an important role in optimizing throughput of secondary transmissions while protecting the quality of service (QoS) of primary users (PUs). In recent years, there has been increasing research attention to spectrum sensing. Several spectrum sensing schemes such as match-filter detection, energy detection [1], [2], cyclostationary detection, and sequential probability ratio test (SPRT) [3], [4] have been proposed. Among these sensing schemes, energy detection is particularly appealing since it does not require any deterministic knowledge on primary signals, a so-called non-coherent detector, and has fairly low implementation complexity. However, energy detection entails a large amount of sensing time especially at low detection signal-to-noise ratio (SNR) levels.

As an alternative to energy detection, the sequential shifted chi-square test (SSCT) was recently proposed in [5]. Like energy detection, SSCT is also a form of non-coherent detector. SSCT is able to achieve comparable detection error performance to energy detection with a much reduced average sample number (ASN). Nevertheless, in SSCT, the test statistic is compared with two predetermined thresholds on every received sample, thus requiring a large number of threshold comparisons particularly at a low SNR level, e.g., the average comparison number ( $\mathrm{ACN})$ is the same as ASN. Additionally, in many scenarios of practical interest, it can be difficult or even infeasible to perform threshold comparison operations based on each received signal sample, particularly when the sampling rate is high and/or SNR is low. To overcome this shortcoming, we propose block-wise SSCT (B-SSCT), in which the received signal samples are parsed into blocks and threshold comparisons are performed at the end of each block. Thus, the number of threshold comparisons can be substantially reduced even with a moderate block length. In particular, B-SSCT subsumes, as special cases, energy detection and SSCT. Like the original SSCT and energy detection, B-SSCT is a form of non-coherent detector, e.g., it does not require any deterministic knowledge on primary signals. Numerical algorithms are applied to evaluate false-alarm and miss-detection probabilities and the ASN of B-SSCT, in a recursive fashion. Simulation and numerical results show that compared with the original SSCT, B-SSCT is capable of achieving almost the same detection error performance with a significantly reduced ACN and a slightly increased ASN. An implementation example demonstrates potential practical feasibility of B-SSCT in a real environment.

\section{System Model AND PREliminaries}

In this section, we start by introducing statistical formulation of the spectrum sensing problem in a narrow-band CR system with a single secondary user (SU), and then give a brief overview on energy detection and SSCT.

\section{A. System Model}

Consider a narrow-band CR communication system with a single SU. The SU shares the same spectrum with a single PU and needs to detect the presence/absence of the PU. The detection of the primary signals is formulated as a binary hypothesis testing problem as

$$
\begin{aligned}
& H_{0}: r_{i}=w_{i}, i=1,2, \ldots, \\
& H_{1}: r_{i}=h s_{i}+w_{i}, i=1,2, \ldots,
\end{aligned}
$$

where $r_{i}$ is the signal received by the $\mathrm{SU}$ at time instant $i, w_{i}$ is additive white Gaussian noise, $s_{i}$ is the transmitted signal of the PU, and $H_{0}$ and $H_{1}$ denote the null and alternative hypotheses, respectively. We further assume that A1) $w_{i} \mathrm{~s}$ are modelled as independent and identically distributed (i.i.d.) complex Gaussian random variables (RVs) with means zero and variances $\sigma_{w}^{2}$, i.e., $w_{i} \sim \mathcal{C N}\left(0, \sigma_{w}^{2}\right)$, A2) the primary signal samples $s_{i}$ are i.i.d. with $\left.E\left(\left|s_{i}\right|^{2}\right)=\sigma_{s}^{2}, \mathrm{~A} 3\right) w_{i}$ and $s_{i}$ are statistically independent, A4) the perfect knowledge on 
the noise variance $\sigma_{w}^{2}$ is available at the SU, and A5) the channel gain $h$ between the primary transmitter (Tx) and the secondary receiver $(\mathrm{Rx})$ is perfectly known at the secondary $\mathrm{Rx}$ and remains unchange during the sensing period.

\section{B. Energy Detection}

In energy detection, the energy of the received signal samples is first computed and then is compared to a predetermined threshold. The test procedure of energy detection is given as

$$
T(\boldsymbol{r})=\sum_{i=1}^{N}\left|r_{i}\right|^{2} \underset{H_{0}}{\stackrel{H_{1}}{\gtrless}} \mathcal{T},
$$

where $\boldsymbol{r}:=\left[r_{1}, r_{2}, \ldots, r_{N}\right], T(\boldsymbol{r})$ denotes the test statistic, and $\mathcal{T}$ denotes the threshold for energy detection.

It follows directly from the central limit theorem (CLT) that as $N$ approaches infinity, the distribution of $T(\boldsymbol{r})$ converges to a normal distribution given as follows [6] $T(\boldsymbol{r}) \mid H_{0} \sim$ $\mathcal{N}\left(N \sigma_{w}^{2}, N \sigma_{w}^{4}\right)$ and $T(\boldsymbol{r}) \mid H_{1} \sim \mathcal{N}\left(N\left(1+\gamma_{m}\right) \sigma_{w}^{2}, N(1+\right.$ $\left.\left.2 \gamma_{m}\right) \sigma_{w}^{4}\right)$ ), where $\gamma_{m}$ denotes the minimum detection SNR and is a design parameter. We assume that the operating SNR is at least equal to $\gamma_{m}$. To distinguish these two different SNRs, we denote by $\gamma_{o}$ the operating SNR.

Let $\bar{P}_{\mathrm{FA}}^{e d}$ and $\bar{P}_{\mathrm{MD}}^{e d}$ be target false-alarm and miss-detection probabilities respectively. Typically, the required sample size $N$ is determined by the target $\bar{P}_{\mathrm{FA}}^{e d}$ and $\bar{P}_{\mathrm{MD}}^{e d}$. Let $N_{e d}^{\min }$ be the minimum sample number required to achieve the target $\bar{P}_{\mathrm{FA}}^{e d}$ and $\bar{P}_{\mathrm{MD}}^{e d}$ at SNR level $\gamma_{m}$. As shown in [1], we have

$$
N_{\min }^{e d}=\left\lceil\gamma_{m}^{-2}\left[\mathcal{Q}^{-1}\left(\bar{P}_{\mathrm{FA}}^{e d}\right)-\mathcal{Q}^{-1}\left(1-\bar{P}_{\mathrm{MD}}^{e d}\right) \sqrt{2 \gamma_{m}+1}\right]^{2}\right\rceil
$$

where $\lceil x\rceil$ denotes the smallest integer not less than $x, \mathcal{Q}(\cdot)$ is the complementary cumulative distribution function of the standard normal RV, i.e., $\mathcal{Q}(x):=(2 \pi)^{-1 / 2} \int_{x}^{\infty} e^{-t^{2} / 2} d t$, and $\mathcal{Q}^{-1}(\cdot)$ denotes its inverse function. It is evident from (3) that for energy detection, the number of required sensing samples is inversely proportional to $\gamma_{m}^{2}$, and thus becomes considerably large when $\gamma_{m}$ is small. As clear from the above description, one major drawback of energy detection is that at a low detection SNR level, it requires a large amount of sensing time to achieve low detection error probabilities [7].

\section{The Original SSCT}

Aiming to achieve reduced sensing time, a non-coherent sequential spectrum sensing scheme, called SSCT, was proposed in [5]. In SSCT, the test statistic is given by $\Lambda_{m}=$ $\sum_{i=1}^{m}\left(\left|r_{i}\right|^{2}-\Delta_{o}\right)$, where $\Delta_{o}$ is a predetermined constant. Assume that the detector needs to make a decision within $M$ received signal samples. The following testing procedure was proposed in [5]

$$
\begin{aligned}
& \text { Reject } H_{0}: \\
& \text { if } \Lambda_{m} \geq b_{o} \text { and } m \leq M-1 \text {, or if } \Lambda_{M} \geq c_{o} ; \\
& \text { Accept } H_{0}: \\
& \text { if } \Lambda_{m} \leq a_{o} \text { and } m \leq M-1 \text {, or if } \Lambda_{M}<c_{o} ; \\
& \text { Continue } \text { Sensing : } \\
& \text { if } \Lambda_{m} \in\left(a_{o}, b_{o}\right) \text { and } m \leq M-1
\end{aligned}
$$

where $a_{o}, b_{o}$, and $c_{o}$ are three predetermined thresholds with $a_{o}<0, b_{o}>0$, and $c_{o} \in\left(a_{o}, b_{o}\right)$.

As can be seen from the procedure (4)-(6), the test statistic is compared with two predetermined thresholds $a_{o}$ and $b_{o}$ on every receive signal sample, thus requiring a large number of threshold comparison operations. To overcome this shortcoming, we propose an extension of SSCT, called B-SSCT, as described in the following section.

\section{B-SSCT: BLOCKWISE SSCT}

In block-wise SSCT, the received signal samples are parsed into a block of length $L$ and threshold comparisons are performed at the end of each block, as shown in Fig. 1. The test statistic at the $p$ th block is computed as follows:

$$
\Xi_{p}=\sum_{i=1}^{p \times L}\left(\left|r_{i}\right|^{2}-\Delta\right), p=1,2, \ldots
$$

where $\Delta$ is a predetermined constant that satisfies $\sigma_{w}^{2}<\Delta<$ $\sigma_{w}^{2}\left(1+\gamma_{m}\right)$ and $p$ is the block index. Assuming that the detector needs to make a decision within $P$ blocks, we now propose the following test procedure:

$$
\begin{aligned}
& \text { Reject } H_{0}: \\
& \qquad \text { if } \Xi_{p} \geq b \text { and } p \leq P-1 \text { or if } \Xi_{P} \geq c ; \\
& \text { Accept } H_{0}: \\
& \text { if } \Xi_{p} \leq a \text { and } p \leq P-1 \text { or if } \Xi_{P}<c ;
\end{aligned}
$$

Continue Sensing :

$$
\text { if } \Xi_{p} \in(a, b) \text { and } p \leq P-1
$$

where $a, b$, and $c$ are three predetermined thresholds with $a<$ $0, b>0$, and $a<c<b$, and $P$ is the truncated block number of the test.

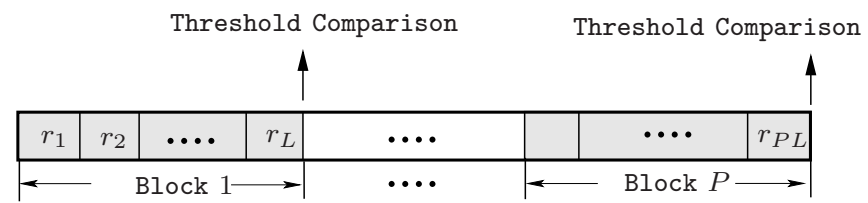

Fig. 1. The B-SSCT spectrum sensing scheme.

Clearly, the test statistic and the test procedure in B-SSCT are quite similar to those in the original SSCT. However, as can be seen from (8)-(10), the truncated sample number in B-SSCT is $P \times L$, and the threshold comparison is only performed every $L$ samples. Hence, the ACN in B-SSCT is $L$ times less than that in the original SSCT. Note that when $L=1$, B-SSCT is nothing but the original SSCT, while when $L=N_{\text {min }}^{e d}$ and $P=1$, B-SSCT is simply energy detection. Thus, it is easy for B-SSCT to meet different design requirements by simply tuning parameters $L$ and $P$.

As depicted in Fig. 2, the test region of the procedure consists of an upper-boundary, the horizonal line $b$, and a lower-boundary, the horizonal line $a$. It is evident from (8)(10) that the test statistic depends only on the amplitude squares of the received signal samples and the constant $\Delta$. 


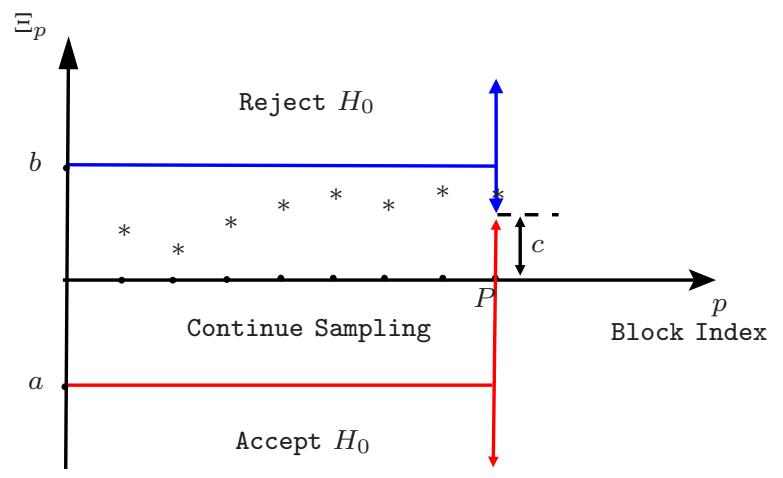

Fig. 2. The test region of B-SSCT.

As will be seen in Section VI, the choice of the constant $\Delta$ depends on $\gamma_{m}$ instead of $\gamma_{o}$, which is typically difficult to obtain in practice.

The thresholds $a, b$, and $c$, the parameter $\Delta$, the block length $L$, and the truncated block number $P$ are selected beforehand, based on an off-line trial and error method. That is, if an initial guess of these parameters does not lead to desirable detection performance, another guess will be made. Such process continues until a desirable detection performance is obtained. In this search process, the critical issue is how to efficiently and accurately evaluate false-alarm and missdetection probabilities and ASN for given thresholds and parameters, as will be addressed in the following two sections.

\section{FAlSE-Alarm AND Miss-DeteCtion PROBABILITIES}

We now turn to the evaluation of the false-alarm probability, $P_{\mathrm{FA}}$, and the miss-detection probability, $P_{\mathrm{MD}}$. Defining $u_{q}=\sum_{l=\bar{p}}^{L}\left(\left|r_{(q-1) L+l}\right|^{2}-\Delta\right)$, we can rewrite $\Xi_{p}$ in (7) as $\Xi_{p}=\sum_{q=1} u_{q}$. Based on the central limit theorem (CLT), the distributions of $u_{q}$ under hypotheses $H_{0}$ and $H_{1}$ can be approximated as $u_{q} \mid H_{0} \sim \mathcal{N}\left(L\left(\sigma_{w}^{2}-\Delta\right), L \sigma_{w}^{4}\right)$ and $u_{q} \mid H_{1} \sim \mathcal{N}\left(L\left(\left(1+\gamma_{m}\right) \sigma_{w}^{2}-\Delta\right), L\left(1+2 \gamma_{m}\right) \sigma_{w}^{4}\right)$.

To compute $P_{\mathrm{FA}}$ and $P_{\mathrm{MD}}$, we resort to a recursive computational method developed in [8]. Clearly, we can write the probability density function (PDF) of $u_{q}$ under $H_{0}$ as

$$
p_{H_{0}}\left(u_{q}\right)=\frac{1}{\sqrt{2 \pi L \sigma_{w}^{4}}} \exp \left(-\frac{\left(u_{q}-L\left(\sigma_{w}^{2}-\Delta\right)\right)^{2}}{2 L \sigma_{w}^{4}}\right)
$$

and the PDF of $u_{q}$ under $H_{1}$ as

$$
\begin{aligned}
p_{H_{1}}\left(u_{q}\right) & =\frac{\left(1+2 \gamma_{m}\right)^{-\frac{1}{2}}}{\sqrt{2 \pi L \sigma_{w}^{4}}} \times \\
& \exp \left(-\frac{\left(u_{q}-L\left(\left(1+\gamma_{m}\right) \sigma_{w}^{2}-\Delta\right)\right)^{2}}{2\left(1+2 \gamma_{m}\right) L \sigma_{w}^{4}}\right) .
\end{aligned}
$$

Evaluating $P_{\mathrm{FA}}$ and $P_{\mathrm{MD}}$ follow quite similar procedures as follows. Without loss of generality, we will only show how to evaluate $P_{\mathrm{MD}}$. Recall that $P$ is the maximum number of blocks to observe. Denote $\Xi_{P-k}$ by $t_{k}$. Let $G_{k}\left(t_{k}\right)$ denote the miss-detection probability of B-SSCT conditioning on the fact that the first $(P-k)$ blocks have been observed, the present value $t_{k}=\Xi_{P-k}$, and the test statistics have not crossed either boundary in the previous $(P-k-1)$ blocks. If $a<t_{k}<b$, an additional sample (the $(P-k+1)$ th block) is needed. Let $u$ be the next observed value of $u_{q}$. The conditional probability $G_{k}\left(t_{k} \mid u\right)$ can be readily obtained as

$$
G_{k}\left(t_{k} \mid u\right)= \begin{cases}0 & \text { if } u>b-t_{k} \\ 1 & \text { if } u<a-t_{k} \\ G_{k-1}\left(t_{k}+u\right) & \text { if } a-t_{k} \leq u \leq b-t_{k} .\end{cases}
$$

Using (13), we can recursively compute $G_{k}\left(t_{k}\right)$ as

$$
\begin{aligned}
& G_{k}\left(t_{k}\right)= \\
& \int_{-\infty}^{a-t_{k}} p_{H_{1}}(u) d u+\int_{a-t_{k}}^{b-t_{k}} G_{k-1}\left(t_{k}+u\right) p_{H_{1}}(u) d u
\end{aligned}
$$

for $k=1, \cdots, P$ with the following initial condition:

$$
G_{0}\left(t_{0}\right)=0, \text { if } t_{0} \geq c ; \quad G_{0}\left(t_{0}\right)=1, \text { otherwise. }
$$

Employing the above recursive process, we can obtain $G_{P}(0)$, which is equal to the miss-detection probability, $P_{\mathrm{MD}}$. By using the same procedure, the false-alarm probability $P_{\mathrm{FA}}$ can be evaluated by making corresponding changes in (14) and (15).

\section{Average Comparison Number and Average SAMPLE NUMBER}

In this section, we evaluate the ACN and the ASN for BSSCT. Clearly, the ASN is simply equal to $L$ times ACN. Hence, we will only focus on the evaluation of the ACN. The number of threshold comparisons required to yield a decision is a RV, which we denote by $P_{s}$. Let $E(\cdot)$ denote the expectation operation. The ACN is simply equal to $E\left(P_{s}\right)$ and can be expressed as

$$
E\left(P_{s}\right)=E_{H_{0}}\left(P_{s}\right) P\left(H_{0}\right)+E_{H_{1}}\left(P_{s}\right) P\left(H_{1}\right)
$$

where $E_{H_{i}}\left(P_{s}\right)$ denotes the ACN conditioned on $H_{i}$ and $P\left(H_{i}\right)$ denotes the probability of hypothesis $H_{i}$ for $i=0,1$. Since $1 \leq P_{s} \leq P$, we can express $E_{H_{i}}\left(P_{s}\right)$ as

$$
E_{H_{i}}\left(P_{s}\right)=\sum_{N=1}^{P} N P_{H_{i}}\left(P_{s}=N\right), i=0,1
$$

where $P_{H_{i}}\left(P_{s}=N\right)$ is the conditional probability that the test ends at the $N$ th block under $H_{i}$. In other words, $P_{H_{i}}\left(P_{s}=N\right)$ is the probability of the event that the test statistic first crosses either lower- or upper-boundary at block $N$ under $H_{i}$.

Let $\mathcal{C}_{N}$ denote the event that the test statistics $\left(\Xi_{1}, \ldots, \Xi_{N}\right)$ do not cross either the upper boundary $b$ or the lower boundary $a$, i.e., $\Xi_{p} \in(a, b)$ for $p=1, \ldots, N$. For notational convenience, let us define $\mathcal{C}_{0}$ as a universe set, i.e., $P_{H_{i}}\left(\mathcal{C}_{0}\right)=1$ for $i=0,1$. Clearly, we have

$$
\begin{aligned}
& P_{H_{i}}\left(P_{s}=N\right) \stackrel{(a)}{=} P_{H_{i}}\left(\mathcal{C}_{N-1}\right)-P_{H_{i}}\left(\mathcal{C}_{N}\right), N \in[1, P-1] \\
& P_{H_{i}}\left(P_{s}=P\right) \stackrel{(b)}{=} P_{H_{i}}\left(\mathcal{C}_{P-1}\right), N=P
\end{aligned}
$$

where the two terms $P_{H_{i}}\left(\mathcal{C}_{N-1}\right)$ and $P_{H_{i}}\left(\mathcal{C}_{N}\right)$ on the righthand side (RHS) of the equality $(a)$ in (18) are the probabilities 
TABLE I

COMPARISONS AMONG B-SCCT, SSCT AND ENERGY DETECTION

\begin{tabular}{c|c|c|c|c}
\hline$\gamma_{m}(\mathrm{~dB})$ & -5 & -10 & -15 & -20 \\
\hline$L$ & 10 & 38 & 178 & 550 \\
$c$ & -9.5 & -20 & -50 & -70 \\
$b$ & 56 & 345 & 2500 & 21000 \\
$\Delta$ & 3.66 & 10.5 & 32.1 & 100.5 \\
\hline$P_{\mathrm{FA}}$ (B-SSCT, Monte Carlo) & 0.057 & 0.103 & 0.153 & 0.202 \\
$P_{\mathrm{FA}}$ (B-SSCT, Numerical) & 0.053 & 0.102 & 0.152 & 0.199 \\
$P_{\mathrm{FA}}$ (SSCT, Monte Carlo) & 0.056 & 0.103 & 0.154 & 0.201 \\
$P_{\mathrm{FA}}$ (ED, Monte Carlo) & 0.055 & 0.101 & 0.150 & 0.200 \\
\hline$P_{\mathrm{MD}}$ (B-SSCT, Monte Carlo) & 0.049 & 0.099 & 0.151 & 0.202 \\
$P_{\mathrm{MD}}$ (B-SSCT, Numerical) & 0.051 & 0.101 & 0.152 & 0.204 \\
$P_{\mathrm{MD}}$ (SSCT, Monte Carlo) & 0.048 & 0.100 & 0.156 & 0.203 \\
$P_{\mathrm{MD}}$ (ED, Monte Carlo) & 0.047 & 0.096 & 0.149 & 0.200 \\
\hline ASN (B-SSCT, Monte Carlo) & 102 & 542 & 3381 & 22814 \\
ASN (B-SSCT, Numerical) & 102 & 542 & 3381 & 22820 \\
ACN (B-SSCT, Monte Carlo) & 10 & 14 & 19 & 41 \\
ASN (ACN) (SSCT, Monte Carlo) & 94 & 509 & 3154 & 21875 \\
$N_{\text {min }}^{\text {ed }}$ & 140 & 722 & 4450 & 28600 \\
\hline $\mathcal{E}_{\text {B-SSCT }}$ & $27 \%$ & $25 \%$ & $24 \%$ & $20 \%$ \\
$\mathcal{E}_{\text {SSCT }}$ & $32 \%$ & $30 \%$ & $29 \%$ & $24 \%$ \\
\hline \multicolumn{2}{|c|}{}
\end{tabular}

of the events that under $H_{i}$, the test statistic does not cross either of two boundaries before or at the $(N-1)$ th block and before or at the $N$ th block respectively for $N=1, \ldots, P-1$, and the term on the RHS of the equality $(b)$ in (19) denotes the probability of the event that under $H_{i}$, the test statistic does not cross either boundary before or at the $(P-1)$ th block.

Substituting (18) and (19) in (17), we can rewrite (17) as

$$
E_{H_{i}}\left(P_{s}\right)=1+\sum_{N=1}^{P-1} P_{H_{i}}\left(\mathcal{C}_{N}\right) .
$$

We next need to evaluate $P_{H_{i}}\left(\mathcal{C}_{N}\right)$. Since evaluating $P_{H_{1}}\left(\mathcal{C}_{N}\right)$ and evaluating $P_{H_{0}}\left(\left(\mathcal{C}_{N}\right)\right.$ follow the same procedure, we will only describe how to evaluate $P_{H_{1}}\left(\mathcal{C}_{N}\right)$ in the sequel. Instead of evaluating $P_{H_{1}}\left(\mathcal{C}_{N}\right)$ directly, we compute $P_{H_{1}}\left(\mathcal{C}_{N}^{c}\right)$ by performing a procedure similar to the one in calculating the miss-detection probability. Note that $\mathcal{C}_{N}^{c}$ denotes the event that the test procedure given in (8)-(10) terminates before or at the $N$ th block, i.e., the test statistic crosses either the upper or lower boundary before or at the $N$ th block. According to (15), $G_{k}\left(t_{k}\right)$ also depends on $c$. With a slight abuse of notation, we rewrite $G_{k}\left(t_{k}\right)$ as $G_{k}\left(t_{k}, c\right)$. Let $V_{N}$ denote the event that the test statistic crosses the lower boundary before or at the $N$ th block, and $U_{N}$ denote the event that the test statistic does not cross the upper-boundary before or at the $N$ th block. It is easy to see $P_{H_{1}}\left(V_{N}\right)=G_{N}(0, a)$ and $P_{H_{1}}\left(U_{N}\right)=G_{N}(0, b)$. Obviously, $P_{H_{1}}\left(\mathcal{C}_{N}^{c}\right)$ can be written as $P_{H_{1}}\left(\mathcal{C}_{N}^{c}\right)=G_{N}(0, a)+1-G_{N}(0, b)$ where $G_{N}(t, a)$ and $G_{N}(t, b)$ can be obtained by applying (14) recursively. Hence, we have $P_{H_{1}}\left(\mathcal{C}_{N}\right)=G_{N}(0, b)-G_{N}(0, a)$. According to (20), we have

$$
E_{H_{1}}\left(P_{s}\right)=1+\sum_{N=1}^{P-1}\left(G_{N}(0, b)-G_{N}(0, a)\right) .
$$

Following the same procedure, $E_{H_{0}}\left(P_{s}\right)$ can be evaluated by using $P_{H_{0}}\left(\mathcal{C}_{N}\right)$, which can be evaluated in a similar manner

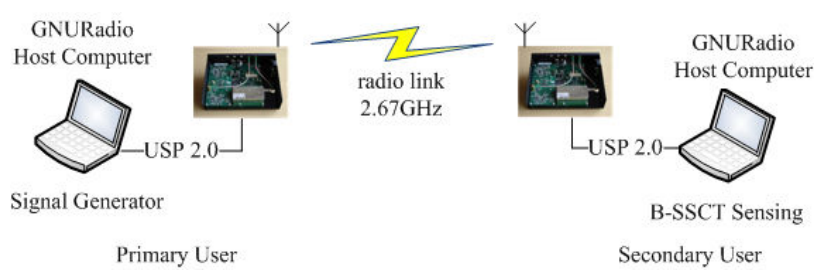

Fig. 3. Spectrum sensing testbed using B-SSCT.

as evaluating $P_{H_{1}}\left(\mathcal{C}_{N}\right)$. Hence, after obtaining $E_{H_{0}}\left(P_{s}\right)$ and $E_{H_{1}}\left(P_{s}\right)$, we can readily evaluate the ACN from (16).

\section{Simulation and Numerical Results}

This section presents simulation and numerical results to demonstrate the effectiveness of B-SSCT. In the following example, we select $\Delta$ to be $\sigma_{w}^{2}\left(1+\gamma_{m} / 2\right)$, which yields $E_{H_{0}}\left(\Xi_{N}\right)=-E_{H_{1}}\left(\Xi_{N}\right)$. The truncated sample sizes are selected to be the minimum sample numbers required by energy detection to achieve specified false-alarm and miss-detection probabilities. The threshold $a$ is always chosen to be $-b$. Following conventional terminology in sequential detection, we define the efficiencies of B-SSCT and SSCT as $\mathcal{E}_{\mathrm{B}-\mathrm{SSCT}}=$ $1-\mathrm{ASN}_{\mathrm{B}-\mathrm{SSCT}} / N_{\min }^{e d}$ and $\mathcal{E}_{\mathrm{SSCT}}=1-\mathrm{ASN}_{\mathrm{SSCT}} / N_{\text {min }}^{e d}$, where $N_{\text {min }}^{e d}$ is determined from (3).

Test Example (Comparisons among B-SSCT, SSCT, and energy detection): Table I shows comparisons among B-SSCT, SSCT, and energy detection in terms of false-alarm and missdetection probabilities, the ASN and the ACN for different $\gamma_{m}$. The truncated sizes corresponding to $\mathrm{SNR}_{m}=-5$, $-10,-15$ and $-20 \mathrm{~dB}$, are selected to be the minimum sample sizes required by energy detection to achieve target $\left(\bar{P}_{\mathrm{FA}}, \bar{P}_{\mathrm{MD}}\right)=(0.05,0.05),(0.1,0.1),(0.15,0.15)$, and $(0.2,0.2)$, respectively. The parameters $b, \Delta$ and $c$ are given in the table. It is shown in Table I that compared with energy detection, B-SSCT can achieve about $20 \% \sim 27 \%$ savings on the ASN while maintaining a comparable detection error performance. In this example, the primary signals are assumed to be Gaussian distributed with mean zero and variance $\sigma_{s}^{2}$. In addition, B-SSCT requires a slightly more (about $5 \%$ more) ASN than SSCT but it needs much less ACN than SSCT. It can be also observed from the table that the false-alarm and missdetection probabilities, as well as the ASN obtained by the recursive algorithm match well with those obtained by Monte Carlo simulations.

\section{An IMPLEMENTATION EXAMPLE}

In order to demonstrate potential practical feasibility of B-SSCT, we next present an implementation example of BSSCT using the spectrum sensing testbed developed in [9]. As depicted in Fig. 3, our spectrum sensing testbed consists of two host computers each running a GNU radio module, and two universal software radio peripheral (USRP) devices each with a RFX 2400 daughterboard. One USRP is used to generate primary signals and the other one is used as a receiver. The USRP hardware is connected to the host computer via a USB 2.0 cable. The testbed is used to sense a frequency band 


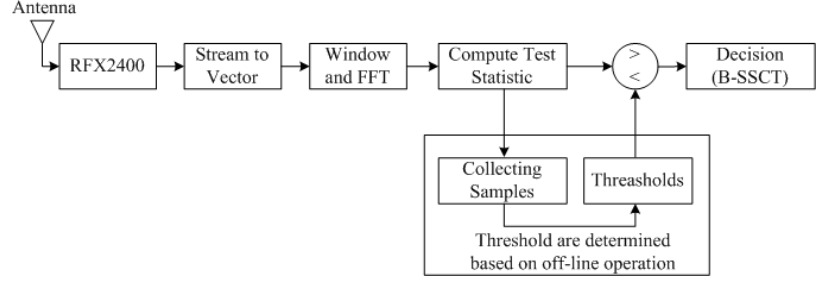

Fig. 4. Block diagram of the received signal detection pathway.

with a central frequency $2.67 \mathrm{GHz}$, which is a portion of the worldwide interoperability for microwave access (WIMAX) licensed band. The proposed B-SSCT method is implemented based on GNU radio 3.3 running on a Ubuntu 9.04 Linux operating system. Interested readers may refer to [9] for a detailed exposition of our testbed.

Fig. 4 depicts the block diagram of the B-SSCT based receiver. The continuous-time received signals are first sampled using an analog-to-digital (A/D) converter located in the RFX 2400 daughterboard. The resulting signal samples are parsed into blocks of length $Q$, where $Q$ is the size of fast Fourier transform (FFT) used in GNU radio. In order to reduce frequency leakage, we apply the Blackman-Harris window to the received signal samples. After the serial-to-parallel (S/P) conversion, the filtered signal samples are then fed into the inputs of FFT. The output signal samples at a fixed FFT bin are collected since the narrow band model is considered in this paper. The test statistic is computed and threshold comparisons are performed at the end of each block of length $L$.

Table II compares the false-alarm and miss-detection probabilities and ASN obtained by the Monte-Carlo simulation and obtained by the experiment using our testbed for B-SSCT. We choose $L=20, \gamma_{o}=-8.9 \mathrm{~dB}$, and $P=30$. In both simulation and experiment, we adopt Gaussian primary signals. In our experiment, the primary Tx and the secondary $\mathrm{Rx}$ are placed very close and the channel between them is a line-of-sight channel. Thus, fading effects are negligible. The sensing frequency band is a part of a licensed WIMAX band, and thus suffers relatively low interferences. The experiment results are averaged over a large number of runs. As can seen from the table, the simulation results and experiment results match well.

\section{CONCLUSIONS AND DISCUSSION}

In this paper, we have proposed an extension of SSCT, namely Block-wise SSCT. Compared with the original SSCT, $\mathrm{B}-\mathrm{SSCT}$ requires a significantly reduced ACN. Besides this enhancement, B-SSCT naturally inherits several desirable attributes from the original SSCT as follows: 1) it has a low implementation complexity; 2) it is a form of non-coherent detection; 3) it requires much shorter sensing time to achieve a comparable detection performance as compared with energy detection; and 4) it offers desirable flexibility in striking a tradeoff between the detection performance and the sensing time when $\gamma_{o}$ mismatches with $\gamma_{m}$. These traits make B-SSCT a practical spectrum sensing scheme for cognitive radio.
TABLE II

Monte-Carlo Simulation Results Versus Experiment Results FOR B-SSCT $\left(L=20, P=30\right.$ AND $\left.\gamma_{o}=-8.9 \mathrm{~dB}\right)$

\begin{tabular}{c|c}
\hline$a$ & -0.0050 \\
$b$ & 0.0050 \\
$c$ & -0.0017 \\
$\Delta$ & $2.92 \times 10^{-4}$ \\
\hline$P_{\mathrm{FA}}$ (Monte Carlo) & 0.101 \\
$P_{\mathrm{FA}}$ (Experiment) & 0.097 \\
\hline$P_{\mathrm{MD}}$ (Monte Carlo) & 0.098 \\
$P_{\mathrm{MD}}$ (Experiment) & 0.096 \\
\hline ASN (Monte Carlo) & 261 \\
ASN (Experiment) & 242 \\
\hline
\end{tabular}

One major challenge in designing B-SSCT is how to determine appropriate parameters including thresholds $a, b$, and $c$, block length $L$, and maximum block number $P$. In this paper, we have proposed a trial-and-error procedure that determines these parameters in an off-line basis. Yet, the procedure may not yield parameters that lead to an optimal or even near optimal detection performance. The issue of how to choose these parameters to optimize detection performance appears much more difficult as compared to one in the energy detection case. In this paper, we have made an assumption that the SU has perfect knowledge on the coefficient of the channel between the primary $\mathrm{Tx}$ and the secondary Rx. In practice, certain cooperation between the $\mathrm{SU}$ and the PU is required in order to obtain a good estimation of the channel coefficient. Like energy detection, B-SSCT also suffers from noise power uncertainty. The issues of how sensitive B-SSCT is to channel estimation errors and noise power uncertainty, are currently under investigation.

\section{REFERENCES}

[1] Y.-C. Liang, Y. Zeng, E. Peh, and A. Hoang, "Sensing-throughput tradeoff for cognitive radio networks," IEEE Trans. Wireless Commun., vol. 7 , no. 4, pp. 1326-1337, Apr. 2008.

[2] H. Urkowitz, "Energy detection of unknown deterministic signals," Proc. IEEE, vol. 55, no. 4, pp. 523-531, Apr. 1967.

[3] N. Kundargi and A. Tewfik, "Hierarchical sequential detection in the context of dynamic spectrum access for cognitive radios," in Proc. IEEE 14th Int. Conf. on Electronics, Circuits and Systems, Marrakech, Morocco, Dec. 11-14, 2007, pp. 514-517.

[4] S. J. Kim and G. B. Giannakis, "Rate-optimal and reduced-complexity sequential sensing algorithms for cognitive ofdm radios," in Proc. 43rd Conf. on Info. Sciences and Systems, Johns Hopkins Univ., Baltimore, MD, Mar. 18 - 20, 2009.

[5] Y. Xin, H. Zhang, and S. Rangarajan, "SSCT: A simple spectrum sensing scheme for cognitive radio," in Proc. IEEE Global Communications Conference (GLOBECOM), Honolulu, HI, USA, Nov. 30-Dec.4, 2009.

[6] Z. Quan, S. Cui, and A. Sayed, "Optimal linear cooperation for spectrum sensing in cognitive radio networks," IEEE J. Select. Topics in Signal Processing, vol. 2, no. 1, pp. 28-40, Feb. 2008.

[7] R. Tandra and A. Sahai, "SNR walls for signal detection," IEEE Journal of Selected Topics in Signal Processing, vol. 2, no. 1, pp. 4-17, Feb. 2008.

[8] S. M. Pollock and D. Golhar, "Efficient recursions for truncation of the SPRT," Technical Report No. 85-24, Dept. of Industrial and Operations Engineering, University of Michigan, Aug. 1985.

[9] K. Kim, Y. Xin, and S. Rangarajan, "Energy detection based spectrum sensing for cognitive radio: An experimental study," in Proc. IEEE Global Communications Conference (GLOBECOM), Miami, FL, USA, Dec. 610, 2010. 